\title{
Gene Deletion Algorithms for Minimum Reaction Network Design by Mixed-integer Linear Programming for Metabolite Production in Constraint-based Models: gDel_minRN
}

Takeyuki Tamura ( $\nabla$ tamura@kuicr.kyoto-u.ac.jp )

Kyoto University

Ai Muto-Fujita

RIKEN Center for Biosystems Dynamics Research

Yukako Tohsato

Ritsumeikan University

Tomoyuki Kosaka

Yamaguchi University

\section{Research Article}

Keywords: algorithm, metabolic network, constraint-based model, flux balance analysis, mixed-integer linear programming, growth coupling, gene deletion

Posted Date: July 1st, 2021

DOI: https://doi.org/10.21203/rs.3.rs-611371/v1

License: (9) This work is licensed under a Creative Commons Attribution 4.0 International License.

Read Full License 


\section{RESEARCH}

Gene deletion algorithms for minimum reaction : network design by mixed-integer linear : programming for metabolite production in : constraint-båsed models: gDel_minRN

Takeyuki Tamura $^{{ }^{*}}$, Ai Muto-Fujita ${ }^{2}$, Yukako Tohsato ${ }^{3}$ and Tomoyuki Kosaka ${ }^{4,5}{ }_{11}$ 
Correspondence:

amura@kuicr.kyoto-u.ac.jp

Bioinformatics Center, Institute

or Chemical Research, Kyoto

niversity, Uji, Kyoto, Japan

ull list of author information is

vailable at the end of the article

\section{Abstract}

Background: Genome-scale constraint-based metabolic networks play an important role in the simulation of growth coupling, which means that cell growth and target metabolite production are simultaneously achieved. To achieve growth coupling, a minimal reaction-network-based design is known to be effective. However, the obtained reaction networks often fail to be realized by gene deletions due to conflicts with gene-protein-reaction relations.

Results: Here, we developed gDel_minRN that determines gene deletion strategies using mixed-integer linear programming to achieve growth coupling by repressing the maximum number of reactions via gene-protein-reaction relations. Computational experiments were conducted in which gDel_minRN was applied to iML1515, a genome-scale model of Escherichia coli. The target metabolites were three vitamins that are highly valuable and require cost-effective bioprocesses for economics and the environment. gDel_minRN successfully calculated gene deletion strategies that achieve growth coupling for the production of biotin (vitamin B7), riboflavin (vitamin B2), and pantothenate (vitaminB5).

Conclusion: Since gDel_minRN calculates a constraint-based model of the minimum number of gene-associated reactions without conflict with gene-protein-reaction relations, it helps biological analysis of the core parts essential for growth coupling for each target metabolite. The source codes are implemented in MATLAB, CPLEX, and COBRA Toolbox. The obtained data and source codes are available on $\{\{$ https://github.com/taketam/gDel-minRN $\}\}$

Keywords: algorithm; metabolic network; constraint-based model; flux balance analysis; mixed-integer linear programming; growth coupling; gene deletion

\section{${ }^{25}$ Background}

${ }^{26}$ Computational approaches are becoming increasingly important in the production ${ }^{26}$

${ }^{27}$ of useful metabolites using microorganisms $[1,2,3,4,5,6,7]$. One of the most ${ }^{27}$

${ }^{28}$ popular mathematical models in genome-scale metabolic engineering simulations is ${ }^{28}$

${ }^{29}$ the constraint-based model Constraint-based models mainly consist of metabolic ${ }^{29}$

30

networks and gene-protein-reaction (GPR) networks.

31 Metabolic networks represent the relationship between chemical reactions and ${ }^{31}$

${ }^{32}$ compounds in cells. Many chemical reactions are catalyzed by enzymatic proteins ${ }^{32}$

${ }^{33}$ encoded by genes. Therefore, metabolic networks can be controlled by gene dele- ${ }^{33}$ 
${ }^{1}$ tions through reaction deletions. The relationships between reactions and genes are ${ }^{1}$

${ }^{2}$ represented by GPR networks, in which the relationships between genes, proteins, ${ }^{2}$

${ }^{3}$ and reactions are represented by Boolean functions.

4 In the metabolic network part of the constraint-based model, steady states are ${ }^{4}$ ${ }^{5}$ assumed in which each metabolic reaction rate (flux) is constant. Such an analysis ${ }^{5}$ ${ }^{6}$ is called flux balance analysis (FBA) [8]. In FBA, (1) for each compound, the sum of ${ }^{6}$

${ }^{7}$ the producing fluxes is equal to the sum of the consuming fluxes; (2) in each reaction, ${ }^{7}$ ${ }^{8}$ the fluxes of substrates and products must satisfy the ratio in the chemical reaction ${ }^{8}$ 9 equation, and (3) the upper and lower bounds are given for each flux.

10 The constraint-based model includes a virtual reaction that represents the cell ${ }^{10}$ ${ }^{11}$ growth. The cell growth reaction of the constraint-based model was designed to ${ }^{11}$ ${ }^{12}$ match the results of the biological experiments. In the most standard FBA with ${ }^{12}$ ${ }^{13}$ constraint-based models, cell growth is maximized in the simulation because geno- ${ }^{13}$ ${ }^{14}$ types that result in higher cell growth are more likely to remain in the culture ${ }^{14}$ ${ }^{15}$ after repeated passaging. The cell growth reaction rate and the target metabolite- ${ }^{15}$ ${ }^{16}$ producing reaction rate are called the growth rate $(\mathrm{GR})$ and production rate ${ }^{16}$ ${ }^{17}(\mathbf{P R})$, respectively.

18 Therefore, in the simulation of useful metabolite production by FBA, we often ${ }^{18}$

${ }^{19}$ evaluate PR when GR is maximized. When cell growth and the target metabolite ${ }^{19}$

${ }^{20}$ production co-occur, we say that growth coupling is achieved. However, the num- ${ }^{20}$

${ }^{21}$ ber of metabolites for which growth coupling is achieved under natural conditions ${ }^{21}$

${ }^{22}$ is limited. Therefore, it is often necessary to calculate the gene deletion strategy for ${ }^{22}$

${ }^{23}$ the given constraint-based model and the target metabolite. (See also Sub figure ${ }^{23}$ ${ }^{24} 1 \mathrm{~A}$.)

${ }^{25}$ Among the many existing methods $[9,10,11,12,13,14,15,16]$, one of the most ef- ${ }^{25}$

${ }^{26}$ ficient methods for calculating reaction deletion strategies for growth coupling is the ${ }^{26}$

${ }^{27}$ elementary flux vector-based method [17]. The elementary flux vector-based method ${ }^{27}$

${ }^{28}$ determines a non-decomposable flux distribution that includes the cell growth re- ${ }^{28}$

${ }^{29}$ action and the target metabolite production reaction and deletes the reactions that ${ }^{29}$

${ }^{30}$ are not used by the flux distribution. In other words, this method selects a minimal ${ }^{30}$

${ }^{31}$ number of reactions to be used in the flow where cell growth forces the production ${ }^{31}$

${ }^{32}$ of the target metabolite and deletes reactions that are not used. It was shown that ${ }^{32}$

${ }^{33}$ the elementary flux vector-based method can compute the reaction deletion strate- ${ }^{33}$ 
Figure 1 (A) Problem setting of this study. PR of the target metabolite is evaluated when the GR

2 is maximized. (B) The idea of gDel_minRN algorithm. The maximum number of reactions are

repressed via gene deletions for the growth coupling.

5gies for growth coupling for most target metabolites for E. coli and S. cerevisiaes 6under aerobic conditions by the combination of such core flows [17]. However, fewer6 7than $10 \%$ of the reaction deletion strategies were feasible as gene deletion strategies sbecause of the gene conflicts when the GPR network was considered[18, 19]. 8

9 Therefore, it would be desirable if such a method based on core flow detection for ${ }^{9}$ ${ }^{10}$ reaction deletion strategies could also detect gene deletion strategies. However, it is ${ }^{10}$ ${ }^{11}$ not straightforward to directly extend the calculation of reaction deletion strategies ${ }^{11}$ 12 to the calculation of gene deletion strategies.

13 In this work, to achieve growth coupling by gene deletions, we have developed ${ }^{13}$ ${ }^{14}$ a mixed-integer linear programming (MILP)-based algorithm, gDel_minRN, to ${ }^{14}$ ${ }^{15}$ calculate the gene deletion strategies that inactivate as many reactions as possible ${ }^{15}$ ${ }^{16}$ that are not essential for growth coupling (See also Sub figure 1B). gDel_minRN ${ }^{16}$ ${ }^{17}$ calculates gene deletion strategies that obtain the minimum reaction network for ${ }^{17}$ ${ }^{18}$ growth coupling.

19 We conducted computational experiments on iML1515, a genome-scale constraint- ${ }^{19}$

${ }^{20}$ based model of E. coli, the most common model organism. The target metabolites ${ }^{20}$

${ }^{21}$ were riboflavin (vitamin B2), pantothenate (vitamin B5), and biotin (vitamin B7). ${ }^{21}$

${ }^{22}$ Vitamins have been industrially produced by chemical synthesis and biosynthesis. ${ }^{22}$

${ }^{23}$ Considering the sustainability, biosynthesis is more promising than chemical syn- ${ }^{23}$

${ }^{24}$ thesis, which produces pollutants, and improvement of vitamins biosynthesis is still ${ }^{24}$

${ }^{25}$ needed because high productivity and cost savings are important factors [20]. The ${ }^{25}$

${ }^{26}$ reduction of metabolic pathways leads to efficient vitamin production by reduc- ${ }^{26}$

${ }^{27}$ ing the amount of protein required. When gDel_minRN was applied, gene deletion ${ }^{27}$

${ }^{28}$ strategies for growth coupling were successfully obtained for these three vitamins. ${ }^{28}$

${ }^{29}$ The gene deletion strategies obtained by gDel_minRN do not contradict the GPR ${ }^{29}$

${ }^{30}$ network, and allow us to design metabolic networks that achieve growth coupling of ${ }^{30}$

${ }^{31}$ these compounds by repressing the maximum number of reactions. Therefore, if we ${ }^{31}$

${ }^{32}$ analyze the gene deletion strategies obtained by gDel_minRN, we may be able to ${ }^{32}$

${ }^{33}$ clarify the biological significance of the core part required for growth coupling for ${ }^{33}$ 


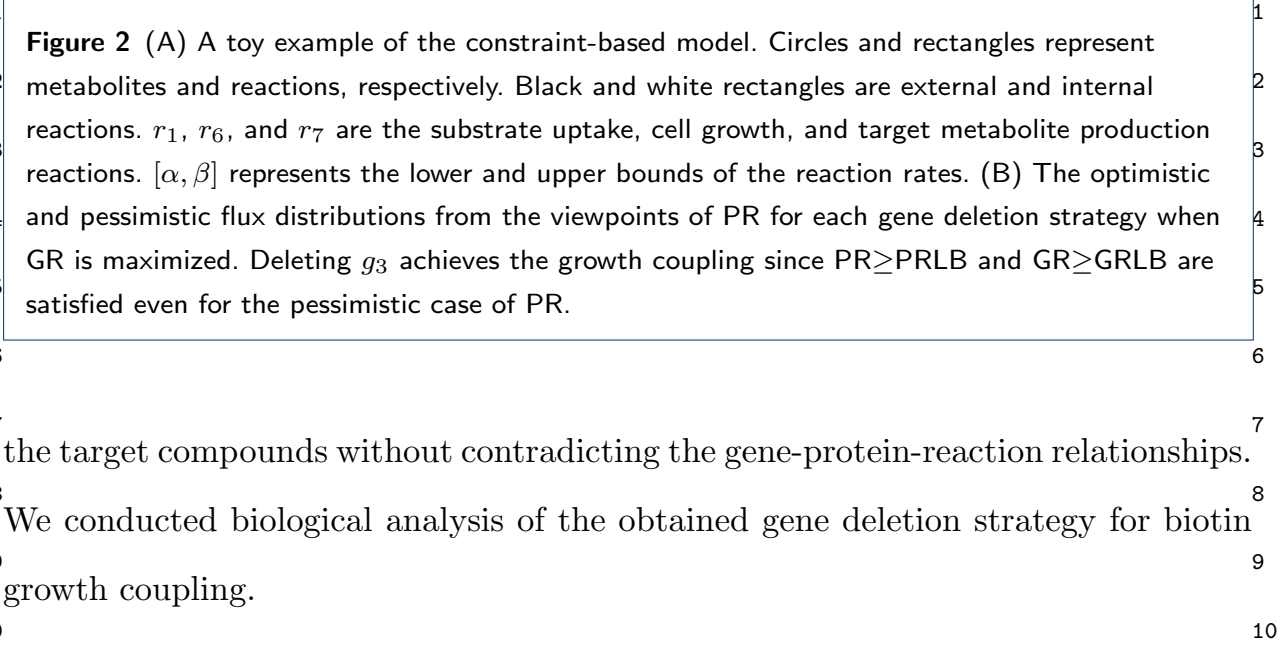

${ }^{11}$ Results

${ }^{12}$ Developed algorithm 12

${ }^{13}$ The developed algorithm gDel_minRN searches, using MILP, the flux and corre- ${ }^{13}$

14 sponding gene deletions that satisfy 14

${ }^{15}$ (1) GR and PR are above the given thresholds, GRLB and PRLB, 15

${ }^{16}(2)$ The number of reactions repressed by gene deletions is maximum, 16

${ }^{17}(3) \mathrm{GR}$ is maximized where (2) has a higher priority than (3). 17

${ }^{18}$ It should be noted that the GR and PR obtained above are not always realized ${ }^{18}$

${ }^{19}$ when GR is maximized without PRLB. Therefore, gDel_minRN tests whether the ${ }^{19}$

${ }^{20}$ obtained gene deletion strategy achieves growth coupling under the condition that ${ }^{20}$

${ }^{21} \mathrm{GR}$ is maximized without PRLB. In particular, gDel_minRN checks the lowest PR ${ }^{21}$

${ }^{22}$ value when GR is maximized. If the obtained gene deletion strategy does not achieve ${ }^{22}$

${ }^{23}$ growth coupling in this pessimistic case, then the gene deletion strategy is added to ${ }^{23}$

${ }^{24}$ the prohibited list and another gene deletion strategy is searched in the same way ${ }^{24}$

${ }^{25}$ by MILP.

${ }^{26}$ For example, suppose that $\mathrm{GRLB}=\mathrm{PRLB}=1$ in Sub figure $2 \mathrm{~A}$. When GR is maxi- ${ }^{26}$

${ }^{27}$ mized under the conditions of $\mathrm{GR} \geq 1$ and $\mathrm{PR} \geq 1$, the flux distribution for each gene ${ }^{27}$

${ }^{28}$ deletion strategy is summarized in Table $1(\mathrm{~A})$. Because deleting $g_{1}, g_{2}$, or $g_{5}$ cannot $^{28}$

${ }^{29}$ satisfy $\mathrm{GR}>\mathrm{GRLB}$ or $\mathrm{PR}>\mathrm{PRLB}$, the gene deletion strategy candidates that can ${ }^{29}$ ${ }^{30}$ satisfy $(1)$ are limited to $\left\{g_{3}\right\},\left\{g_{4}\right\}$ and $\left\{g_{3}, g_{4}\right\}$. The number of repressed reac- ${ }^{30}$

${ }^{31}$ tions by deleting $\left\{g_{3}\right\},\left\{g_{4}\right\}$ and $\left\{g_{3}, g_{4}\right\}$ are 1,0 and 1 , respectively, as shown in ${ }^{31}$

${ }^{32}$ Table $1(\mathrm{~A})$. Therefore, gDel_minRN first selects the deletion of $\left\{g_{3}\right\}$ or $\left\{g_{4}\right\}$. When ${ }^{32}$

${ }^{33}$ the gene deletion strategy is not uniquely determined under the condition that the ${ }^{33}$ 
${ }_{1}$ Table 1 (A) The flux distribution for each gene deletion strategy when GR is maximized under the condition with $G R \geq 1$ and $P R \geq 1$. (B) The priority of each gene deletion candidate and resulting flux ${ }^{2}$ distribution. (A)

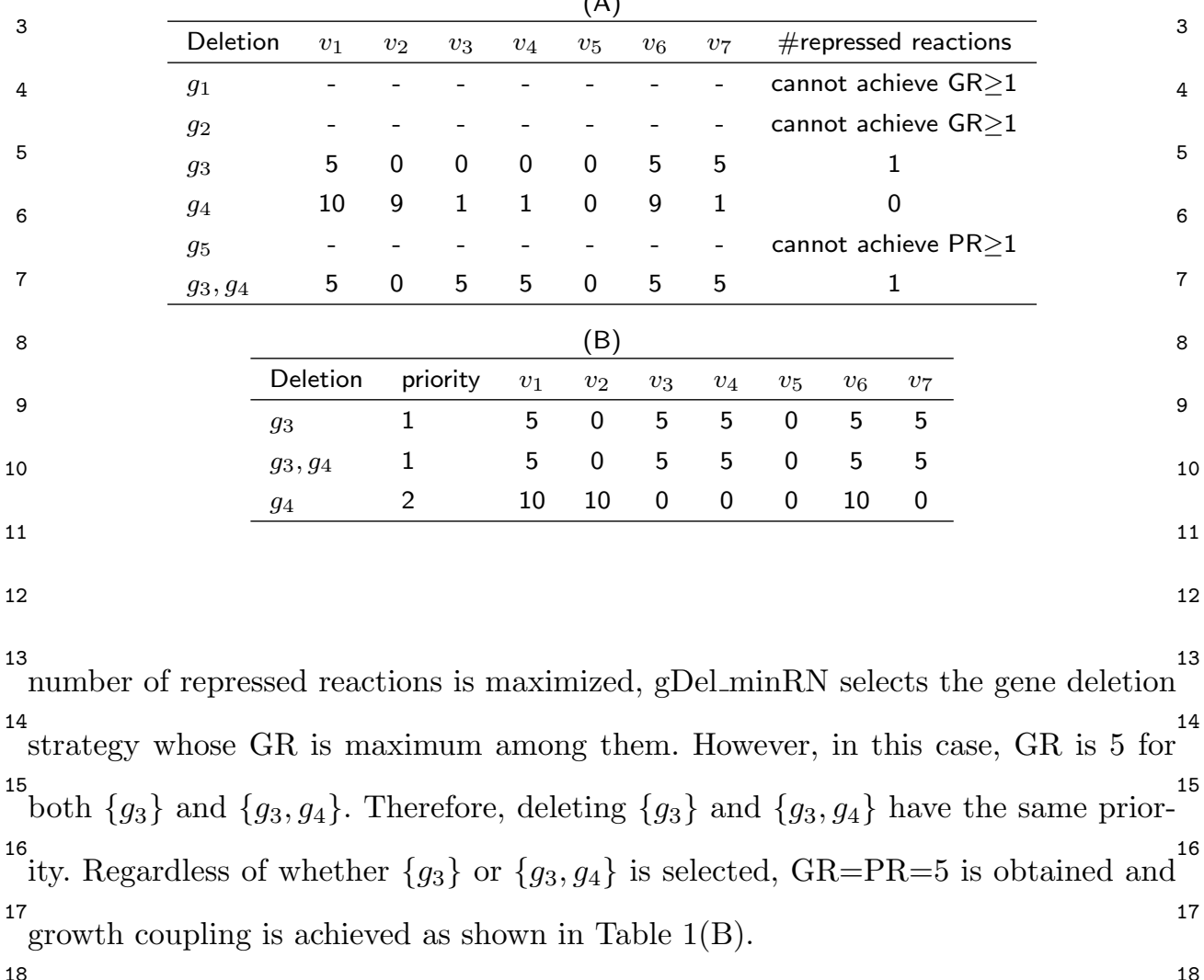

19 gDel_minRN stops if the candidate of the gene deletion strategy achieves growth g $_{19}$ ${ }_{20}$ coupling. If growth coupling is not achieved, the obtained gene deletion strategy is ${ }_{20}$ ${ }_{21}$ added to the prohibited list, and gDel_minRN searches for the next solution. If no so- 21 ${ }_{22}$ lution is obtained after the designated number of iterations, maxloop, gDel_minRN ${ }_{22}$ ${ }_{23}$ Stops. Although the example is simple for illustration, gDel_minRN can be applied $_{23}$ ${ }_{24}$ to complex GPR rules that combine AND and OR function. An AND function ${ }_{24}$ ${ }_{25} y=x_{1} \wedge x_{2} \wedge \cdots \wedge x_{k}$ is converted into the linear constraints $-x_{1}-\ldots-x_{k}+k y \leq 0_{25}$ ${ }_{26}$ and $x_{1}+\cdots+x_{k}-y \leq k-1$. An OR function $y=x_{1} \vee x_{2} \vee \cdots \vee x_{k}$ is converted into ${ }_{26}$ ${ }_{27}$ the linear constraint $x_{1}+\cdots+x_{k}-k y \leq 0$ and $-x_{1}-\cdots-x_{k}+y \leq 0$. gDel_minRN $\mathrm{N}_{27}$ ${ }_{28}$ cannot be applied directly to the case where NOT functions are included, but many $_{28}$ ${ }_{29}$ latest genome-scale models such as iML1515 do not include NOT functions. 29 ${ }^{30}$ In gDel_minRN, we use MILP with PRLB to obtain the candidate for gene dele- ${ }^{30}$ ${ }^{31}$ tion strategies and then test whether growth coupling is achieved during GR max- ${ }^{31}$ ${ }^{32}$ imization without PRLB. The reason why gDel_minRN maximizes the number of ${ }^{32}$ ${ }^{33}$ repressed reactions is that the more similar the flux distributions are when using ${ }^{33}$ 
${ }^{1} \mathrm{PRLB}$ and when not using PRLB, the higher the success rate of the algorithm. This ${ }^{1}$

${ }^{2}$ is also the reason why the second optimization target is the maximization of GR. ${ }^{2}$

3

${ }_{7}$ All procedures in the computational experiments were implemented on a CentOS $7_{7}$ ${ }_{8}$ machine with an Intel Xeon Processor with $2.30 \mathrm{GHz} 18 \mathrm{C} / 36 \mathrm{~T}$, and $128 \mathrm{~GB}$ mem${ }_{9}$ ory. This workstation had CPLEX 12.8, COBRA Toolbox 2021 [21], and MATLAB ${ }_{10}$ R2017b. An auxiliary exchange reaction was temporarily added to the model to ${ }_{10}$ ${ }_{11}$ simulate the target metabolite production.

12 In the computational experiments, three vitamins, pantothenate (vitamin B5),12 13biotin (vitamin B7), and riboflavin (vitamin B2), were used as target metabolites.13 14These three metabolites are highly valuable, but no effective biosynthesis methods14 15have been established. We applied gDel_minRN for growth coupling of these three15 16target metabolites to iML1515[22], which is one of the most recent genome-scale16 17constraint-based models of $E$. coli and includes 1515 genes, 2712 reactions, and 187717 18metabolites. 18 19 Table 2 summarizes the gene deletion strategies obtained using gDel_minRN. Be- ${ }^{19}$ ${ }^{20}$ cause the number of repressed reactions is maximized in gDel_minRN, the average ${ }^{20}$ ${ }^{21}$ number of deleted genes, 960.33 , was almost twice as large as the average num- ${ }^{21}$ ${ }^{22}$ ber of remaining genes, 554.67 . When the obtained gene deletion strategy was ap- ${ }^{22}$ ${ }^{23}$ plied and GR was maximized, the PR ratio to the theoretical maximum (TMPR) ${ }^{23}$ 24 was $0.7444,0.1004$, and 0.1702 , respectively. GR ratio to the theoretical maximum ${ }^{25}$ (TMGR) were $0.2485,0.1702$, and 0.1434 , respectively. Because the minimum re- ${ }^{25}$ ${ }^{26}$ quired PR/TMPR and GR/TMGR were 0.1 in the experiments, we can say that the ${ }^{26}$ ${ }^{27}$ strategies for pantothenate and biotin worked well, but that for riboflavin was not ${ }^{27}$ ${ }^{28}$ sufficient. The maximum computation time was approximately $6 \mathrm{~h}$, which is within ${ }^{28}$ ${ }^{29}$ the acceptable range for individual calculations, but may not be suitable for batch ${ }^{29}$ 30 calculations for hundreds of target metabolites. The first candidate gene deletion 30 ${ }^{31}$ strategy obtained for each target metabolite failed to achieve growth coupling and ${ }^{31}$ ${ }^{32}$ succeeded in the second or third attempt using the prohibited list. Therefore, we can ${ }^{32}$ ${ }^{33}$ say that the iterative search algorithm using the prohibited list worked effectively. ${ }^{33}$ 
${ }_{1}$ Table 2 Three vitamins used as the target metabolites and the summary of the obtained gene deletion strategies by gDel_minRN. An auxiliary exchange reaction was temporarily added to the 2 model to simulate each target metabolite production.

\begin{tabular}{|c|c|c|c|c|c|c|c|}
\hline 3 & Target & \#used genes & $\mathrm{PR} / \mathrm{TMPR}$ & GR/TMGR & time & loop & abb̧̧eviation \\
\hline & Pantothenate (vitamin B5) & 562 & 0.7444 & 0.2485 & $4 \mathrm{~h} 40 \mathrm{~m} 43 \mathrm{~s}$ & 3 & pnto__R_c \\
\hline 4 & Biotin (vitamin B7) & 538 & 0.1004 & 0.1702 & $6 \mathrm{~h} 20 \mathrm{~m} 26 \mathrm{~s}$ & 2 & btn_c \\
\hline 5 & Riboflavin (vitamin B2) & 564 & 0.0437 & 0.1434 & $2 \mathrm{~h} 58 \mathrm{~m} 49 \mathrm{~s}$ & 2 & ribf|v_cc \\
\hline
\end{tabular}

6

\section{${ }_{7}$ Discussion}

8Biological analysis for biotin production

9One of the motivations for developing gDel_minRN was to calculate the core parts9 10required for growth coupling and to biologically elucidate which features are neces-10 ${ }^{11}$ sary for growth coupling and which are not. Among the three gene deletion strate-11 12gies obtained by gDel_minRN, the most genes were deleted in the case of biotin.12 ${ }^{13}$ Therefore, the obtained biotin production pathway was analyzed biologically using13 14Escher [23] and KEGG Mapper [24] as follows.

15 In the obtained pathway for biotin production by gDel_minRN, it was observed ${ }^{15}$

${ }^{16}$ that the pathways from acetyl-CoA to acetate were removed from the map. The ${ }^{16}$ ${ }^{17}$ acetyl-CoA obtained in glycolysis was consumed in the TCA cycle or converted to ${ }^{17}$ ${ }^{18}$ acetate, and was also used to generate malonyl-CoA. Since malonyl-CoA is located ${ }^{18}$

${ }^{19}$ at the beginning of the biotin-generating pathway, we hypothesized that by inhibit- ${ }^{19}$

${ }^{20} \mathrm{ing}$ the conversion of acetyl-CoA to acetate, acetyl-CoA that could not be fully ${ }^{20}$

${ }^{21}$ consumed by the TCA cycle was used for biotin generation via malonyl-CoA. ${ }^{21}$

22 To test this hypothesis, we revived all eight deleted genes (b0871, b2296, b0968, ${ }^{22}$

${ }^{23} \mathrm{~b} 2297$, b2458, b4069, b3588, b1241) located on the pathways from acetyl-CoA to ${ }^{23}$

${ }^{24}$ acetate. As a result, $\mathrm{GR}=0.3341$ and $\mathrm{PR}=0$ were obtained. This reinforces the ${ }^{24}$

${ }^{25}$ hypothesis that by removing the acetyl-CoA to the acetate pathway, the substate ${ }^{25}$

${ }^{26}$ used for cell growth was replaced by biotin production via malonyl-CoA. 26

27 Since the existing basic strategy for improving biotin productivity using bacterial ${ }^{27}$

${ }^{28}$ cells is the overexpression of rate-limiting enzymes, removal of negative regula- ${ }^{28}$

${ }^{29}$ tors and addition of intermediates or precursors [25], complete optimization of the ${ }^{29}$

${ }^{30}$ metabolic pathways by altering the whole genomic network has not been extensively ${ }^{30}$

${ }^{31}$ tested. The constructed pathway for biotin synthesis from iML1515, a recent solid ${ }^{31}$

${ }^{32}$ computational model for E. coli metabolism, with the lowest number of reactions by ${ }^{32}$

${ }^{33}$ gDel_minRN in this study showed new possibilities for the E. coli metabolic pathway ${ }^{33}$ 
${ }^{1}$ that can be changed from the original genome. Although the constructed pathway ${ }^{1}$

${ }^{2}$ is stoichiometrically reasonable because iML1515 has almost complete metabolic ${ }^{2}$

${ }^{3}$ network [22], it is not clear whether it can be created in E. coli real cells. There- ${ }^{3}$

${ }^{4}$ fore, we considered this pathway from a biological point of view. The constructed ${ }^{4}$

${ }^{5}$ pathway from glucose to biotin can be separated into two phases, from glucose to ${ }^{5}$

${ }^{6}$ malonyl acyl-carrier-protein (ACP) and malonyl-ACP to biotin, respectively ( $\mathrm{Sub}^{6}$

${ }^{7}$ figure $3 \mathrm{~A}$ ). For biotin production, S-adenosylmethionine (SAM) and L-alanine are ${ }^{7}$

${ }^{8}$ required to synthesize and adjust the production ratio in the upper pathway to ${ }^{8}$

${ }^{9}$ drive the lower pathway (Sub figure $3 \mathrm{~A}$ ). The reactions in the lower pathway were ${ }^{9}$

${ }^{10}$ not so unique because almost one connected pathway from malonyl-ACP to bi- ${ }^{10}$

${ }^{11}$ otin in E. coli [20]. On the other hand, the biological consideration of the upper ${ }^{11}$

${ }^{12}$ pathway, glucose to malonyl-ACP, revealed three notable characteristics. The most ${ }^{12}$

${ }^{13}$ interesting characteristic was that nicotinamide adenine dinucleotide (NAD) was $^{13}$

${ }^{14}$ not used throughout the reactions. This result probably came from the calculation ${ }^{14}$

${ }^{15}$ conditions for growth coupling with the minimum medium and glucose as the sole ${ }^{15}$

${ }^{16}$ carbon substrate because all amino acids and nucleotides are required for synthesis, ${ }^{16}$

${ }^{17}$ and the enzyme responsible for these reactions utilizes nicotinamide adenine dinu- ${ }^{17}$

${ }^{18}$ cleotide phosphate (NADP) mainly as an electron carrier. In addition, the strategy ${ }^{18}$

${ }^{19}$ of gDel_minRN is to reduce the reactions as possible then if electron carrier $\mathrm{NAD}^{19}$

${ }^{20}$ not used in the pathway the many metabolic reactions can be reduced. Although ${ }^{20}$

${ }^{21}$ it is very outlandish, since the dependency of NAD for biological metabolism is ${ }^{21}$

${ }^{22}$ come from enzyme specificity, if there is no NAD-dependent enzyme, and NADP ${ }^{22}$

${ }^{23}$ can drive all related reactions, NAD is not essential. Therefore, it is not biologically ${ }^{23}$

${ }^{24}$ impossible. The second characteristic is the requirement for aerobic metabolism. ${ }^{24}$

${ }^{25}$ In these reactions, a high amount of NADPH was produced from glucose to ribose ${ }^{25}$

${ }^{26} 5$-phosphate pathway, and oxidation was performed in dihydroxyacetone phosphate ${ }^{26}$

${ }^{27}$ to glycerol 3-phosphate by glycerol-3-phosphate dehydrogenase, and NADP could ${ }^{27}$

${ }^{28}$ then be produced (Sub figure 3B). Countering, in the opposite direction from glyc- ${ }^{28}$

${ }^{29}$ erol 3-phosphate to dihydroxyacetone phosphate utilizing ubiquinone-8 (UQ8) as ${ }^{29}$

${ }^{30}$ an electron acceptor to produce $\mathrm{UQ} 8 \mathrm{H}_{2}$ (Sub figure $3 \mathrm{~B}$ ). In addition, the reactions ${ }^{30}$

${ }^{31}$ for pyruvate to lactate and succinate to fumarate generate $\mathrm{UQ} 8 \mathrm{H}_{2}$. These reac- ${ }^{31}$

${ }^{32}$ tions cause high accumulation of $\mathrm{UQ} 8 \mathrm{H}_{2}$; oxidation is required to proceed with the ${ }^{32}$

${ }^{33}$ metabolic reaction accomplished by using oxygen as an electron acceptor on the ${ }^{33}$ 
Figure 3 The constructed pathway for biotin production. (A) Overview of the biotin synthesis 2 pathway from iML1515 classified into two pathways as upper and lower pathway. (B) Precise flow 2 of upper pathway, from glucose to malonyl-ACP. The number indicated with each arrows shows the flux value of each reaction. The abbreviations are as follows; NADPH, Nicotinamide adenine dinucleotide phosphate reduced form; UQ8, ubiquinone-8; ACP, acyl carrier protein; PEP, phosphoenolpyruvate; OAA, oxaloacetate; PRPP, Phosphoribosyl diphosphate.

6

${ }^{8}$ respiratory chain, which also causes adenosine triphosphate (ATP) production in ${ }^{8}$

${ }^{9}$ respiratory chains. High ATP production also requires not only the lower path- ${ }^{9}$

${ }^{10}$ way but also nucleotide and amino acid synthesis. Therefore, this pathway requires ${ }^{10}$

${ }^{11}$ oxygen or a respiratory oxidative substrate. We did not investigate the effect of ${ }^{11}$

${ }^{12}$ the presence of oxygen on the constructed pathway. Therefore, future experiments ${ }^{12}$

${ }^{13}$ should consider how substrate and culture conditions affect this pathway. The third ${ }^{13}$

${ }^{14}$ characteristic is that the intermediates of this pathway do not consider about the ${ }^{14}$

${ }^{15}$ cytotoxicity. The upper pathway utilizing methylglyoxal as the intermediate from ${ }^{15}$

${ }^{16}$ dihydroxyacetone phosphate to lactate (Sub figure $3 \mathrm{~B}$ ). The methylglyoxal utilizing ${ }^{16}$

${ }^{17}$ pathway is known in 1,2-propanediol producing bacteria but it shows that high ${ }^{17}{ }^{17}$

${ }^{18}$ totoxicity [26]. This pathway is possible but has problems. Several microorganisms ${ }^{18}$

${ }^{19}$ for 1,2-propanediol production consider the pathway to not be exchanged because ${ }^{19}$

${ }^{20}$ of the reduction in growth or production by the pathway [27]. This suggests that ${ }^{20}$

${ }^{21}$ if we try to resolve more cell suitable pathways, we need some trick to avoid using ${ }^{21}$

${ }^{22}$ the pathways from the literature to produce more realistic computational minimum ${ }^{22}$

23 pathway prediction for production. Finally, several problematic points for the con-

${ }^{24}$ struction or reproduction of this pathway in E. coli were found, but the constructed ${ }^{24}$

${ }^{25}$ pathway was almost biologically possible in our consideration. Interestingly, when ${ }^{25}$

${ }^{26}$ using a short and small number of reactions for some material production, cells can ${ }^{26}$

${ }^{27}$ reduce the protein amount, which finally guides more efficient material production ${ }^{27}$

${ }^{28}$ by the cell. The biological consideration of this pathway is only a knowledge base, ${ }^{28}$

${ }^{29}$ and an experimental demonstration of this pathway on a cell should be performed ${ }^{29}$

${ }^{30}$ in the future. To accomplish this, we need additional strategies, such as reduction of ${ }^{30}$

${ }^{31}$ gene numbers for disruption or high number or gene disruption methods at the ge- ${ }^{31}$

${ }^{32}$ nomic scale. Alternatively, the use of semi-synthetic minimal cells is recommended ${ }^{32}$

${ }^{33}$ to prove this pathway. 33 to prove this pathway. 
${ }^{1}$ Comparison with existing computational methods $\quad 1$

${ }^{2}$ In the calculation of gene deletion strategies for growth coupling, it has been nec- ${ }^{2}$ ${ }^{3}$ essary to minimize the number of genes to be deleted in terms of cost and accuracy ${ }^{3}$ ${ }^{4}[28,19]$. However, gDel_minRN maximizes the number of reactions that are re- ${ }^{4}$ ${ }^{5}$ pressed to obtain the core part necessary for growth coupling, so it would rather ${ }^{5}$ ${ }^{6}$ delete as many genes as possible. Therefore, the obtained gene deletion strategies ${ }^{6}$ ${ }^{7}$ are quite different from those obtained using existing methods. Such a gene dele- ${ }^{7}$ ${ }^{8}$ tion strategy is helpful for biological analysis of which part of the constraint-based ${ }^{8}$ ${ }^{9}$ model is necessary for growth coupling but may not be practical for metabolite pro- ${ }^{9}$

${ }^{10}$ duction with current metabolic engineering technology. However, it could be useful ${ }^{10}$

${ }^{11}$ if zero-based DNA synthesis for metabolite production is possible in the future. $\operatorname{In}^{11}$

${ }^{12}$ addition, under conditions where the product is obtained with growth coupling, it ${ }^{12}$

${ }^{13}$ simplifies the actual production process and enables simultaneous production and ${ }^{13}$

${ }^{14}$ cell maintenance in continuous culture.

15 On the other hand, for reaction deletions, the idea of finding a core network for ${ }^{15}$

${ }^{16}$ growth coupling has been studied using elementary flux vector-based methods [17]. ${ }^{16}$

${ }^{17}$ However, because the obtained reaction deletion strategies often conflict with $\mathrm{GPR}^{17}$

${ }^{18}$ networks, it is difficult to extend the reaction deletion strategies to gene deletion ${ }^{18}$

19 strategies [19].

20 A number of mixed-integer linear prom 20

21 proposed for calculating gene or reaction deletion strategies that result in growth

${ }^{22}$ coupling $[1,2,29,30]$. Solving MILP is an NP-complete problem and requires com- ${ }^{22}$ ${ }^{23}$ putation time proportional to the exponential function of the number of reactions. ${ }^{23}$

${ }^{24}$ Many methods that are not limited to MILP have been proposed to speed up the ${ }^{24}$ ${ }^{25}$ computation time by avoiding the optimization of $\mathrm{PR}[9,10,11,12,13,14,15,16]{ }^{25}$

${ }^{26}$ However, to the best of our knowledge, there is no method for calculating the gene ${ }^{26}$

${ }^{27}$ deletion strategy that results in a minimal network for growth coupling. Therefore, ${ }^{27}$

${ }^{28}$ it is difficult to directly compare the performance of gDel_minRN with those of

29 other methods in computational experiments.

30

${ }^{31}$ Conclusion

${ }^{32}$ In this study, we developed gDel_minRN to calculate gene deletion strategies that ${ }^{32}$

${ }^{33}$ repress as many reactions as possible to achieve growth coupling. Computer ex- ${ }^{33}$ 
${ }^{1}$ periments using three vitamins as target compounds showed that we could find ${ }^{1}$

${ }^{2}$ strategies that deleted more than $60 \%$ of all genes. Among them, we biologically ${ }^{2}$

${ }^{3}$ analyzed the gene deletion strategy for biotin production and tested the hypothesis ${ }^{3}$

${ }^{4}$ that deletion of genes in the pathway from acetyl-CoA to acetate replaces substrate ${ }^{4}$

${ }^{5}$ consumption for cell growth with biotin production. Unlike existing biosynthetic ${ }^{5}$

${ }^{6}$ methods for biotin production, the strategy obtained by gDel_minRN is based on $a^{6}$

${ }^{7}$ fundamental modification of the metabolic pathway. Existing computational meth- ${ }^{7}$

${ }^{8}$ ods aim to delete a small number of genes or compute core networks by deleting ${ }^{8}$

${ }^{9}$ reactions, and their purpose is fundamentally different from that of gDel_minRN, ${ }^{9}$

${ }^{10}$ which calculates core networks by gene deletion. Analyzing gene deletion strate- ${ }^{10}$

${ }^{11}$ gies obtained by gDel_minRN is helpful for biological analysis for which parts are ${ }^{11}$

${ }^{12}$ necessary for growth coupling. $\quad 12$

$13 \quad 13$

14Methods 14

${ }^{15}$ Definition $\quad{ }^{15}$

${ }^{16}$ Let $C=(M, R, S, L, U, G, F, P)$ be a constraint-based model, where $M={ }^{16}$

${ }^{17}\left\{m_{1}, \ldots, m_{a}\right\}, R=\left\{r_{1}, \ldots, r_{b}\right\}, G=\left(g_{1}, \ldots, g_{c}\right), F=\left(f_{1}, \ldots, f_{b}\right)$, and $P={ }^{17}$

${ }^{18}\left(p_{1}, \ldots, p_{b}\right)$ are sets of metabolites, reactions, genes, Boolean functions, and the ${ }^{18}$

${ }^{19}$ outputs of $F$, respectively. $R$ always includes one special virtual reaction $r_{\text {growth }}{ }^{19}$

${ }^{20}$ that represents cell growth, and the cell growth flux is represented by $v_{\text {growth }} . S$ is a ${ }^{20}$

${ }^{21}$ stoichiometry matrix, where $S_{i j}=k$ means that $r_{j}$ produces $k$ of $m_{i}$ per unit time. ${ }^{21}$

${ }^{22}$ If $k$ is a negative number, then $m_{i}$ is consumed. Let $V=\left\{v_{1}, \ldots, v_{b}\right\}$ be a set of $^{22}$

${ }^{23}$ reaction rates per unit time (flux) of $R$. Let $L=\left\{l_{1}, \ldots, l_{b}\right\}$ and $U=\left\{u_{1}, \ldots, u_{b}\right\}^{23}$

${ }^{24}$ be the sets of the lower and upper bounds for $V$, respectively. 24

${ }^{25} C_{1}=(M, R, S, L, U)$ is called the metabolic network part of $C$. $v_{\text {growth }}$ is called ${ }^{25}$

${ }^{26}$ the growth rate (GR). In FBA using $C_{1}$, GR is maximized by the following linear ${ }^{26}$

${ }^{27}$ programming $(\mathrm{LP})$ : $\quad 27$

28

maximize

$29 \quad 29$

30 so 30

$31 \quad \Sigma_{j} S_{i j} v_{j}=0$ for all $i \quad 31$

${ }^{32} l_{j} \leq v_{j} \leq u_{j}$ for all $j=32$

33 
${ }^{1}$ If the $i$ th column of $S$ has only one non-zero element; in other words, $r_{i}$ connects to ${ }^{1}$

${ }^{2}$ only one metabolite, then $r_{i}$ is called an external reaction, and is considered to be ${ }^{2}$

${ }^{3}$ connected to the external environment. Reactions that are not exchange reactions ${ }^{3}$

${ }^{4}$ are called internal reactions. The flux of the external reaction producing the ${ }^{4}$

${ }^{5}$ target metabolite under the condition that cell growth is maximized is called the ${ }^{5}$

${ }^{6}$ production rate $(\mathbf{P R})$. 6

7 In contrast, $C_{2}=(G, F, P)$ is called the GPR network part of $C$, and $\quad 7$

8

$9 \quad p_{i}=f_{i}\left(g_{i, 1}, \ldots, g_{i, k_{i}}\right)$, where $p, g \in\{0,1\}$ and $1 \leq k_{i} \leq c . \quad 9$

${ }_{11}$ If $p_{i}=0$, then $l_{i}$ and $u_{i}$ are forced to be 0 . In other words,

18

$D \subset G$

19 such that minimizes

20

$v_{\text {target }}$

such that maximizes

$\{g=0$ if $\mathrm{g} \in D+29$

$g=1$, otherwise 30

$v_{\text {target }} \geq P R_{\text {threshold }}$

$v_{\text {growth }} \geq G R_{\text {threshold }}$

$p, g \in\{0,1\}$ 
4Example for problem setting

$$
i=\{1, \ldots, a\}, j=\{1, \ldots, b\}
$$

2

4

5Sub figure $2 \mathrm{~A}$ shows a small toy example of the constraint-based model, where $M=5$

${ }_{8}^{7}\left\{m_{1}, \ldots, m_{4}\right\}, R=\left\{r_{1}, \ldots, r_{7}\right\}$, and $S=\left(\begin{array}{ccccccc}1 & -1 & -1 & 0 & -1 & 0 & 0 \\ 0 & 1 & 0 & 1 & 0 & -1 & 0 \\ 0 & 0 & 1 & -1 & 0 & 0 & 0 \\ 0 & 0 & 0 & 1 & 1 & 0 & -1\end{array}\right)_{9}^{6}$

Because $[\alpha, \beta]$ attached to $r_{i}$ means that $\alpha \leq v_{i} \leq \beta, L=\left\{l_{1}, \ldots, l_{7}\right\}$ and $U={ }_{10}$ ${ }_{11}\left\{u_{1}, \ldots, u_{7}\right\}$ are as follows; $l_{1}, \ldots, l_{7}=0, u_{1}, \ldots, u_{3}=10, u_{4}, u_{5}=5, u_{6}, u_{7}=10{ }_{11}$ ${ }_{12}$ For $C_{2}$, it is given that $G=\left\{g_{1}, \ldots, g_{5}\right\}, F=\left\{f_{1}, \ldots, f_{7}\right\}$ and

$p_{1}=f_{1}=\phi$,

$p_{2}=f_{2}=g 1 \wedge g 2 \wedge g 3$

14

$p_{3}=f_{3}=\phi$,

$p_{4}=f_{4}=g 2 \wedge g 5$,

$p_{5} \quad=f_{5}=(g 3 \vee g 4) \wedge g 5$,

$p_{6}=f_{6}=\phi$,

$p_{7}=f_{7}=\phi$.

${ }^{19}$ Note that $f$ represents a Boolean function, whereas $p$ takes either 0 or $1 . p_{i}=f_{i}=\phi^{19}$

20

${ }^{20}$ means that $r_{i}$ cannot be repressed via gene deletions.

20

${ }^{21}$ In the original state, when GR $\left(v_{6}\right)$ is maximized, all fluxes from $r_{1}$ flow through ${ }^{21}$ ${ }^{22} r_{2}$ to $r_{6}$. Therefore, $v_{1}=v_{2}=v_{6}=10$ and $v_{3}=v_{4}=v_{5}=v_{7}=0$ are obtained as ${ }^{22}$ ${ }^{23}$ shown in the second row of Sub figure $2 \mathrm{~B}$.

${ }^{24}$ If $g_{1}$ is deleted, then $p_{2}=g_{1} \wedge g_{2} \wedge g_{3}=0$ since $g_{1}=0$. Therefore, $r_{2}$ does not $^{24}$ ${ }^{25}$ work and $v_{2}$ is forced to be zero. Similarly, $r_{3}$ does not work and $v_{3}$ is forced to be ${ }^{25}$ ${ }^{26}$ zero because $p_{3}=g_{1}=0$ holds. Therefore, when GR is maximized, fluxes from $r_{1}{ }^{26}$ ${ }^{27}$ cannot reach $r_{6}$, and GR becomes 0 . In the optimistic case for PR, $v_{1}=v_{5}=v_{7}=5^{27}$ ${ }^{28}$ is obtained, but no flux flows in the pessimistic case, as shown in the third and ${ }^{28}$ ${ }^{29}$ fourth rows of Sub figure $2 \mathrm{~B}$, respectively. To ensure the growth coupling, we need ${ }^{29}$ ${ }^{30}$ to evaluate the pessimistic case for PR, and the maximized GR must exceed the ${ }^{30}$ ${ }^{31}$ minimum required value. Therefore, we consider that growth coupling cannot be ${ }^{31}$ ${ }^{32}$ achieved by deleting $g_{1}$. When $g_{2}$ is deleted, similar results are obtained because ${ }^{32}$ 33 neither $r_{2}$ nor $r_{4}$ works. 
1 If $g_{3}$ is deleted, $r_{2}$ does not work but the other reactions can work. Therefore, the ${ }^{1}$ ${ }^{2}$ maximum GR is five because $0 \leq v_{4} \leq 5$. In the optimistic case, the flux from $r_{1}{ }^{2}$ ${ }^{3}$ flows to $r_{7}$ via $r_{5}$ in addition to via $r_{3}$ and $r_{4}$. In this case, $\mathrm{GR}=5$ and $\mathrm{PR}=10$ is ${ }^{3}$

${ }^{4}$ obtained. However, in the pessimistic case, $\mathrm{GR}=\mathrm{PR}=5$ were obtained as shown in ${ }^{4}$

${ }^{5}$ the seventh and eighth rows of Sub figure $2 \mathrm{~B}$, respectively. 5

${ }^{6}$ If $g_{4}$ is deleted, $p_{i}=1$ for all $i$. Therefore, $v_{1}=v_{2}=v_{6}=10$ and $v_{3}=v_{4}=v_{5}={ }^{6}$ ${ }^{7} v_{7}=0$ are obtained when GR is maximized. If $g_{5}$ is deleted, neither $r_{4}$ nor $r_{5}$ works ${ }^{7}$ ${ }^{8}$ since $p_{4}=p_{5}=0$. However, a similar result is obtained because $r_{2}$ works as shown ${ }^{8}$ ${ }^{9}$ in the ninth and tenth rows of Sub figure $2 \mathrm{~B}$, respectively.

10 Suppose that $\mathrm{GRLB}=\mathrm{PRLB}=1$; that is, the minimum required $\mathrm{GR}$ and $\mathrm{PR}$ are 1 . $^{10}$

${ }^{11}$ Then, deleting $g_{3}$ achieves growth coupling because $\mathrm{GR}=\mathrm{PR}=5$ is obtained even for ${ }^{11}$

${ }^{12}$ the pessimistic case and $\mathrm{GR} \geq \mathrm{GRLB}$ and $\mathrm{PR} \geq \mathrm{PRLB}$ are satisfied. In this example, ${ }^{12}$

${ }^{13}$ growth coupling can be achieved by deleting one gene $g_{3}$. However, in practice, it ${ }^{13}$

${ }^{14}$ may be necessary to examine all genes on and off, which results in a combinatorial ${ }^{14}$ explosion.

16

${ }^{17}$ Pseudo code $\quad{ }^{17}$

${ }^{18}$ The pseudo code of gDel_minRN is as follows. 18

${ }^{19}$ Procedure gDel minRN(model, target Met, PRLB, GRLB, maxloop) 19

20

/*Calculating the theoretical maximum production rate.*/ 20

21

$T M P R=\max v_{\text {target }}$

22

23

24

25

26 s.t. $\Sigma_{j} S_{i, j} \cdot v_{j}=0$ for all $1 \leq i \leq a$ $L B_{j} \leq v_{j} \leq U B_{j}$ for all $1 \leq j \leq b$

if $T M P R<P R L B$

return "no solution"

/*Calculating the theoretical maximum growth rate.*/

$T M G R=\max v_{\text {growth }}$

s.t. $\Sigma_{j} S_{i, j} \cdot v_{j}=0$ for all $1 \leq i \leq a$ $L B_{j} \leq v_{j} \leq U B_{j}$ for all $1 \leq j \leq b \quad 29$

* Finding a gene deletion strategy candidate.*/ 30

prohibited_list $=\phi$, loop $=1$

$\max v_{\text {growth }}+T M G R \cdot K O /{ }^{*}$ first maximize \#repressed reactions.*/ 
s.t. $\Sigma_{j} S_{i, j} \cdot v_{j}=0$

$\left\{\begin{array}{l}v_{j}=0 \text { if } p_{j}=0 \\ l_{j} \leq v_{j} \leq u_{j}, \text { otherwise }\end{array}\right.$

$K O$ : the number of repressed reactions $\left(p_{j}=0\right)$.

$\left\{\begin{array}{l}g=0 \text { if } g \in D / *^{*} D \text { is flexible. } * / \\ g=1, \text { otherwise }\end{array}\right.$

else 


\section{${ }^{1}$ List of abbreviations}

${ }^{2}$ Gene-Protein-Reaction (GPR)

${ }^{3}$ Flux Balance Analysis (FBA)

${ }^{4}$ Growth Rate (GR)

${ }^{5}$ Production Rate $(\mathrm{PR})$

${ }^{6}$ Mixed-Integer Linear Programming (MILP)

${ }^{7}$ Theoretical Maximum Production Rate (TMPR)

${ }^{8}$ Theoretical Maximum Growth Rate (TMGR)

${ }^{9}$ Acyl-Carrier-Protein (ACP)

${ }^{10}$ Nicotinamide Adenine Dinucleotide (NAD)

${ }^{11}$ Nicotinamide Adenine Dinucleotide Phosphate (NADP)

${ }^{12}$ Ubiquinone (UQ)

${ }^{13}$ Adenosine Triphosphate (ATP)

${ }^{14}$ Linear Programming (LP)

15

${ }^{16}$ Declarations

17 Ethics approval and consent to participate

Not applicable.

18

${ }_{19}$ Consent for publication

Not applicable.

20

${ }_{21}$ Availability of data and materials

The datasets generated and/or analysed during the current study are available in the GitHub repository,

22 https://github.com/taketam/gDel-minRN

23

Competing interests

${ }^{24}$ The authors declare that they have no competing interests. 24

25

Funding

${ }^{26}$ TT, AMF, YT, and TK were partially supported by grants from JSPS, KAKENHI \#20H04242. No funding body 27 played any roles in the design of the study and collection, analysis, and interpretation of data and in writing the manuscript.

28

${ }_{29}$ Authors' contributions

TT designed this work, developed the algorithm, implemented the software, and conducted the computational

30 experiments. AMF performed the analysis using the database. YT performed the visualization analysis. TK provided 30

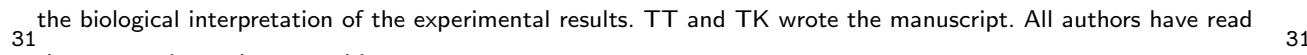
the manuscript and approved it.

32

${ }_{33}$ Acknowledgements

We would like to thank all the editors and reviewers involved in this paper.
3 
${ }^{1}$ Author details 1

$2^{1}$ Bioinformatics Center, Institute for Chemical Research, Kyoto University, Uji, Kyoto, Japan. ${ }^{2}$ Center for 2

Biosystems Dynamics Research, RIKEN, Suita, Osaka, Japan. ${ }^{3}$ Faculty of Information Science and Engineering,

${ }^{3}$ Ritsumeikan University, Kusatsu, Shiga, Japan. ${ }^{4}$ Research Center for Thermotolerant Microbial Resources 3

4 (RCTMR), Yamaguchi University, Yoshida, Yamaguchi, Japan. ${ }^{5}$ Graduate School of Sciences and Technology for 4

Innovation, Yamaguchi University, Yoshida, Yamaguchi, Japan.

5

References

6 1. Burgard, A.P., Pharkya, P., Maranas, C.D.: Optknock: a bilevel programming framework for identifying gene 6

$7 \quad$ knockout strategies for microbial strain optimization. Biotechnology and bioengineering 84(6), 647-657 (2003) 7

2. Pharkya, P., Burgard, A.P., Maranas, C.D.: Optstrain: a computational framework for redesign of microbial

8 production systems. Genome research 14(11), 2367-2376 (2004) 8

3. Pharkya, P., Maranas, C.D.: An optimization framework for identifying reaction activation/inhibition or

9 elimination candidates for overproduction in microbial systems. Metabolic engineering 8(1), 1-13 (2006)

10 4. Patil, K.R., Rocha, I., Förster, J., Nielsen, J.: Evolutionary programming as a platform for in silico metabolic 10 engineering. BMC bioinformatics 6(1), 308 (2005)

11 5. Ranganathan, S., Suthers, P.F., Maranas, C.D.: Optforce: an optimization procedure for identifying all genetic 11

12 manipulations leading to targeted overproductions. PLoS Comput Biol 6(4), 1000744 (2010) 12

6. Rocha, I., Maia, P., Evangelista, P., Vilaça, P., Soares, S., Pinto, J.P., Nielsen, J., Patil, K.R., Ferreira, E.C.,

13 Rocha, M.: Optflux: an open-source software platform for in silico metabolic engineering. BMC systems biology 13 4(1), 1-12 (2010)

147 . Toya, $\mathrm{Y}$, Shimizu, $\mathrm{H}$ : Flux analysis and metabolomics for systematic metabolic engineering of microorganisms. 14

15 Biotechnology advances 31(6), 818-826 (2013) 15

8. Orth, J.D., Thiele, I., Palsson, B. Ø.: What is flux balance analysis? Nature biotechnology 28(3), 245-248

16 (2010)

17 9. Lun, D.S., Rockwell, G., Guido, N.J., Baym, M., Kelner, J.A., Berger, B., Galagan, J.E., Church, G.M.: 17 Large-scale identification of genetic design strategies using local search. molecular systems biology 5(1), 296

18 (2009)

19 10. Rockwell, G., Guido, N.J., Church, G.M.: Redirector: designing cell factories by reconstructing the metabolic 19 objective. PLoS Comput Biol 9(1), 1002882 (2013)

2011. Yang, L., Cluett, W.R., Mahadevan, R.: Emilio: a fast algorithm for genome-scale strain design. Metabolic 20

21 engineering 13(3), 272-281 (2011)

12. Egen, D., Lun, D.S.: Truncated branch and bound achieves efficient constraint-based genetic design

22 Bioinformatics 28(12), 1619-1623 (2012) 22

13. Lewis, N.E., Hixson, K.K., Conrad, T.M., Lerman, J.A., Charusanti, P., Polpitiya, A.D., Adkins, J.N.,

23 Schramm, G., Purvine, S.O., Lopez-Ferrer, D., et al.: Omic data from evolved e. coli are consistent with 23

24 computed optimal growth from genome-scale models. Molecular systems biology 6(1), 390 (2010) 24

14. Gu, D., Zhang, C., Zhou, S., Wei, L., Hua, Q.: Idealknock: a framework for efficiently identifying knockout

25 strategies leading to targeted overproduction. Computational biology and chemistry 61, $229-237$ (2016) 25

26 15. Ohno, S., Shimizu, H., Furusawa, C.: Fastpros: screening of reaction knockout strategies for metabolic 26 engineering. Bioinformatics 30(7), 981-987 (2014)

2716. Tamura, T.: Grid-based computational methods for the design of constraint-based parsimonious chemical 27

28 reaction networks to simulate metabolite production: Gridprod. BMC bioinformatics 19(1), 325 (2018)

17. von Kamp, A., Klamt, S.: Growth-coupled overproduction is feasible for almost all metabolites in five major 28

29 production organisms. Nature communications 8, 15956 (2017) 29

18. Machado, D., Herrgård, M.J., Rocha, I.: Stoichiometric representation of gene-protein-reaction associations

30 leverages constraint-based analysis from reaction to gene-level phenotype prediction. PLoS computational 30

31 biology 12(10), 1005140 (2016) 31

19. Razaghi-Moghadam, Z., Nikoloski, Z.: Genereg: A constraint-based approach for design of feasible metabolic

32 engineering strategies at the gene level. Bioinformatics (2020) 32

33 20. Acevedo-Rocha, C., Gronenberg, L., Mack, M., Commichau, F., Genee, H.: Microbial cell factories for the 33 sustainable manufacturing of b vitamins. Curr. Opin. Biotechnol. 56, 18-29 (2019) 
${ }^{1}$ 21. Heirendt, L., Arreckx, S., Pfau, T., Mendoza, S.N., Richelle, A., Heinken, A., Haraldsdóttir, H.S., Wachowiak, 1

2 J., Keating, S.M., Vlasov, V., et al.: Creation and analysis of biochemical constraint-based models using the 2 cobra toolbox v. 3.0. Nature protocols 14(3), 639-702 (2019)

322. Monk, J.M., Lloyd, C.J., Brunk, E., Mih, N., Sastry, A., King, Z., Takeuchi, R., Nomura, W., Zhang, Z., Mori, 3 H., et al:: iml1515, a knowledgebase that computes escherichia coli traits. Nature biotechnology 35(10), 904-908 (2017)

523. King, Z.A., Dräger, A., Ebrahim, A., Sonnenschein, N., Lewis, N.E., Palsson, B.O.: Escher: a web application 5 for building, sharing, and embedding data-rich visualizations of biological pathways. PLoS Comput Biol 11(8), $1004321(2015)$

724. Kanehisa, M., Sato, Y.: Kegg mapper for inferring cellular functions from protein sequences. Protein Science 7 29(1), 28-35 (2020)

${ }^{8}$ 25. Xiao, F., Wang, H., Shi, Z., Huang, Q., Huang, L., Lian, J., Cai, J., Xu, Z.: Multi-level metabolic engineering 8 of Pseudomonas mutabilis atcc31014 for efficient production of biotin. Metab Eng 61, $406-415$ (2020)

26. Booth, I., Ferguson, G., Miller, S., Li, C., Gunasekera, B., Kinghorn, S.: Bacterial production of methylglyoxal:

10 a survival strategy or death by misadventure. Biochem Soc Trans 31(Pt 6), 1406-1408 (2003) 10

11 27. Niu, W., Kramer, L., Mueller, J., Liu, K., Guo, J.: Metabolic engineering of Escherichia coli for the de novo 11 stereospecific biosynthesis of 1,2-propanediol through lactic acid. Metab Eng Commun 8, 00082 (2019)

1228. Apaolaza, I., Valcarcel, L.V., Planes, F.J.: gmcs: fast computation of genetic minimal cut sets in large 12 networks. Bioinformatics 35(3), 535-537 (2019)

13 29. Kim, J., Reed, J.L., Maravelias, C.T.: Large-scale bi-level strain design approaches and mixed-integer

14 programming solution techniques. PLoS One 6(9), 24162 (2011)

30. Tepper, N., Shlomi, T.: Predicting metabolic engineering knockout strategies for chemical production:

15 accounting for competing pathways. Bioinformatics 26(4), 536-543 (2010) 


\section{Figures}

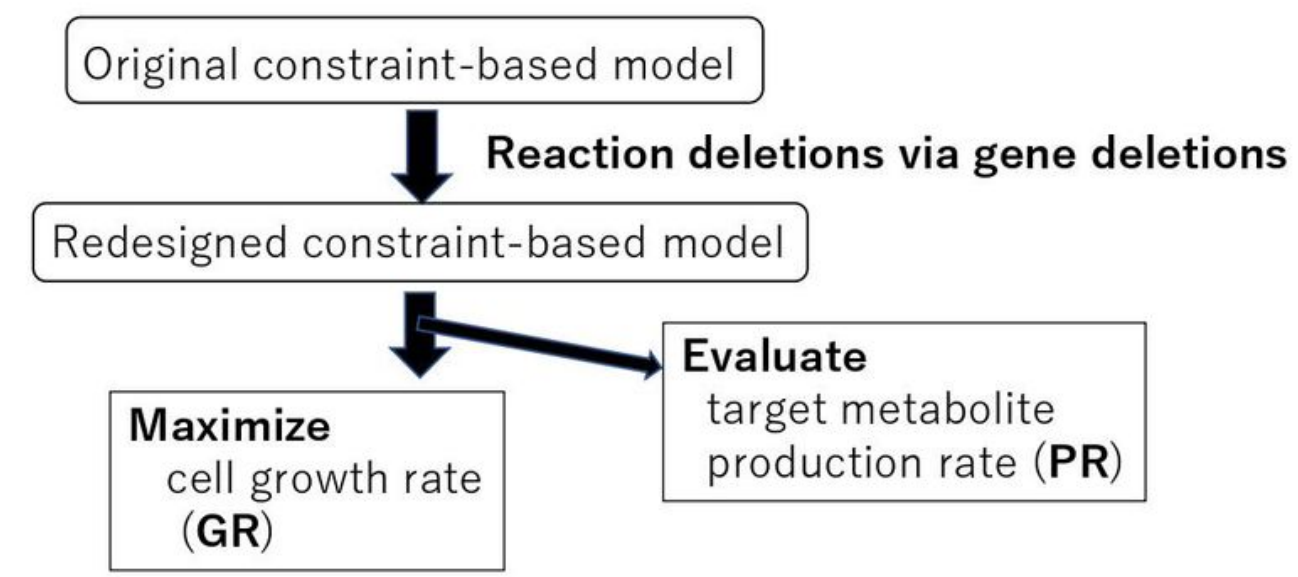

\section{Constraint-based model \\ $=$ metabolic network part + GPR network part}

\section{(A)}

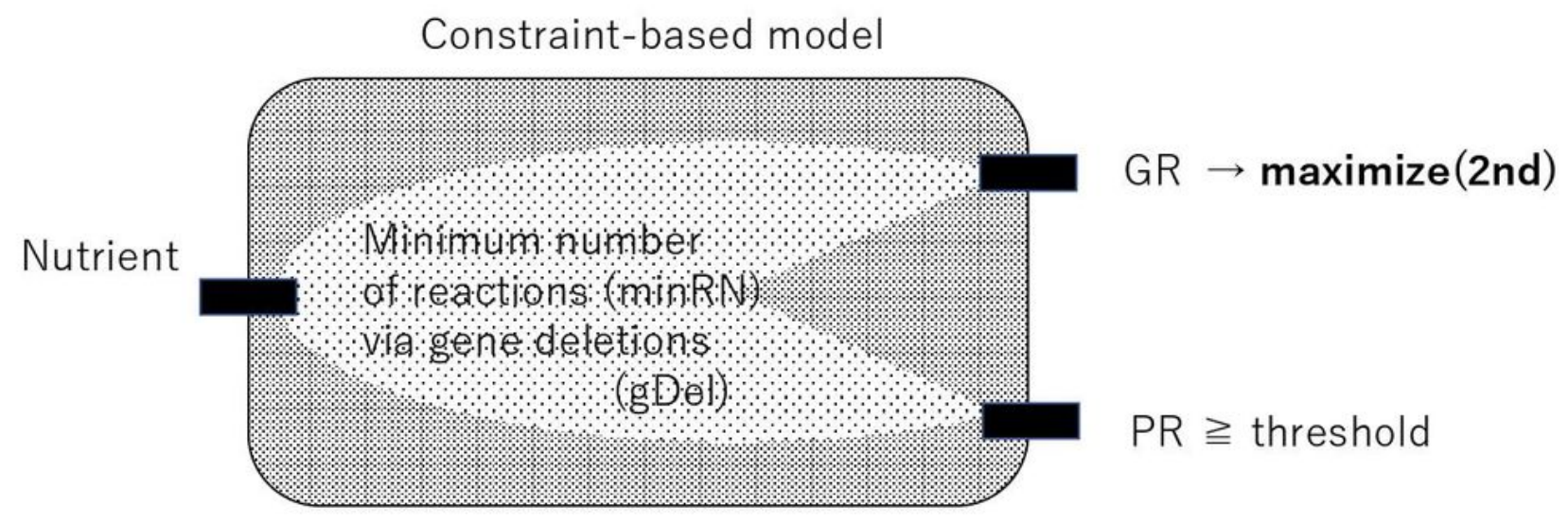

- The effective part that achieves growth coupling.

The unnecessary part to be deleted.

$\rightarrow$ maximize(1st) the number of suppressed reactions

(B)

\section{Figure 1}

(A) Problem setting of this study. PR of the target metabolite is evaluated when the GR is maximized. (B) The idea of gDel minRN algorithm. The maximum number of reactions are repressed via gene deletions for the growth coupling. 


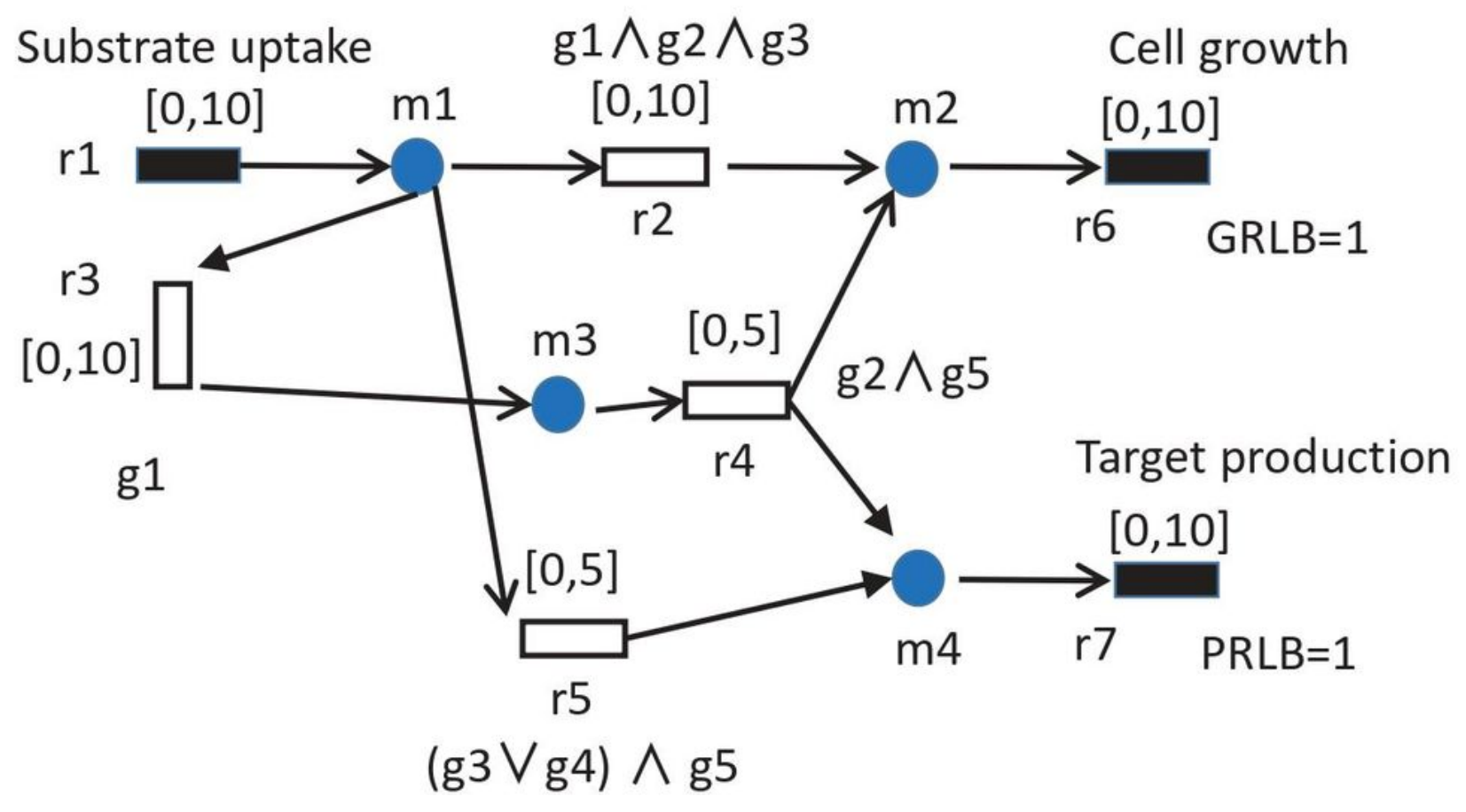

(A)

\begin{tabular}{lcccccccc}
\hline Gene KO & & $v_{1}$ & $v_{2}$ & $v_{3}$ & $v_{4}$ & $v_{5}$ & $v_{6}$ & $v_{7}$ \\
\hline none & both & 10 & 10 & 0 & 0 & 0 & 10 & 0 \\
\hline g1 & best & 5 & 0 & 0 & 0 & 5 & 0 & 5 \\
& worst & 0 & 0 & 0 & 0 & 0 & 0 & 0 \\
\hline g2 & best & 5 & 0 & 0 & 0 & 5 & 0 & 5 \\
& worst & 0 & 0 & 0 & 0 & 0 & 0 & 0 \\
\hline g3 & best & 10 & 0 & 5 & 5 & 5 & 5 & 10 \\
& worst & 5 & 0 & 5 & 5 & 0 & 5 & 5 \\
\hline g4 & both & 10 & 10 & 0 & 0 & 0 & 10 & 0 \\
\hline g5 & both & 10 & 10 & 0 & 0 & 0 & 10 & 0 \\
\hline g1, g2 & both & 0 & 0 & 0 & 0 & 0 & 0 & 0 \\
\hline$\vdots$ & $\vdots$ & $\vdots$ & $\vdots$ & $\vdots$ & $\vdots$ & $\vdots$ & $\vdots$ & $\vdots$ \\
\hline
\end{tabular}

(B)

Figure 2

(A) A toy example of the constraint-based model. Circles and rectangles represent metabolites and reactions, respectively. Black and white rectangles are external and internal reactions. $r 1, r 6$, and $r 7$ are the substrate uptake, cell growth, and target metabolite production reactions. [a, $\beta$ ] represents the lower and upper bounds of the reaction rates. (B) The optimistic and pessimistic flux distributions from the 
viewpoints of $\mathrm{PR}$ for each gene deletion strategy when $\mathrm{GR}$ is maximized. Deleting g3 achieves the growth coupling since $P R \geq P R L B$ and $G R \geq G R L B$ are satisfied even for the pessimistic case of PR.

A

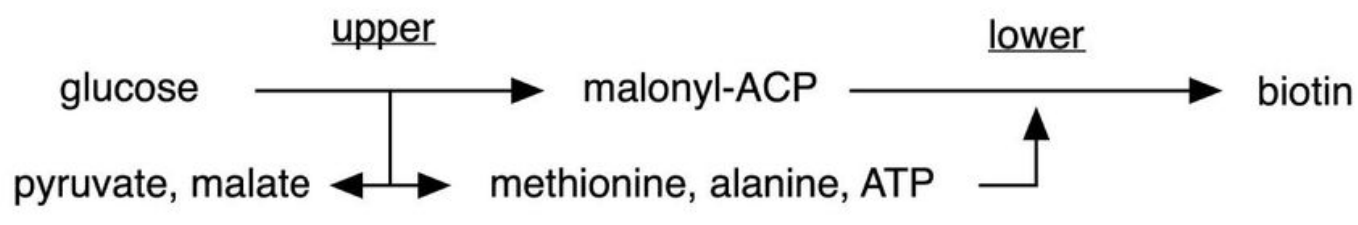

[methionine + adenosine(ATP) --> S-adenosyl methionine (SAM)]

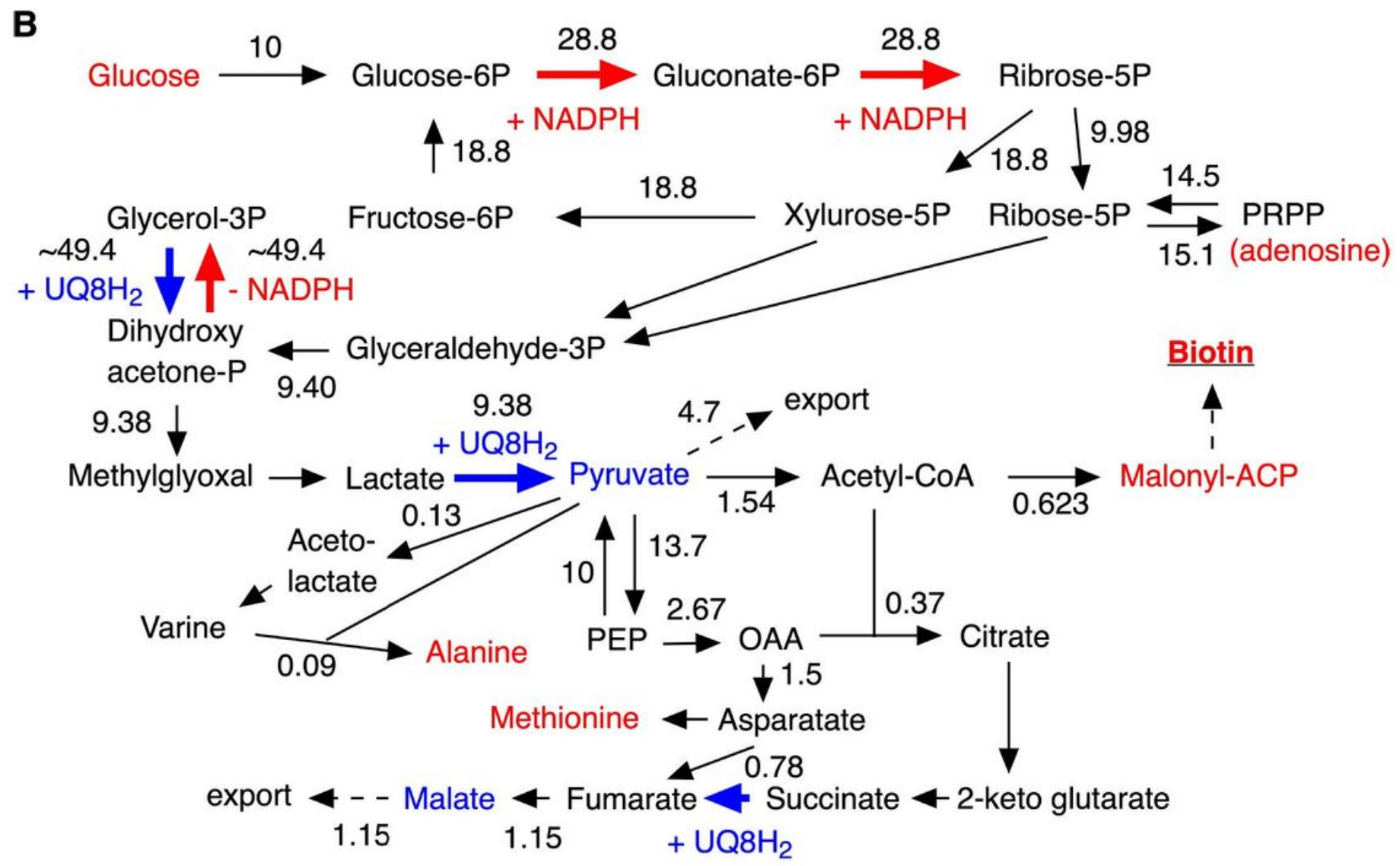

Figure 3

The constructed pathway for biotin production. (A) Overview of the biotin synthesis pathway from iML1515 classified into two pathways as upper and lower pathway. (B) Precise flow of upper pathway, from glucose to malonyl-ACP. The number indicated with each arrows shows the flux value of each reaction. The abbreviations are as follows; NADPH, Nicotinamide adenine dinucleotide phosphate reduced form; UQ8, ubiquinone-8; ACP, acyl carrier protein; PEP, phosphoenolpyruvate; OAA, oxaloacetate; PRPP, Phosphoribosyl diphosphate. 